\title{
Characterizing Weak Chaos using Time Series of Lyapunov Exponents
}

\author{
R.M. da Silva ${ }^{1}$, C. Manchein ${ }^{1}$, M.W. Beims ${ }^{2,3}$, E.G. Altmann ${ }^{3}$ \\ ${ }^{1}$ Departamento de Física, Universidade do Estado de Santa Catarina, 89219-710 Joinville, Brazil \\ ${ }^{2}$ Departamento de Física, Universidade Federal do Paraná, 81531-980 Curitiba, Brazil and \\ ${ }^{3}$ Max-Planck-Institute for the Physics of Complex Systems, \\ Nöthnitzer Str. 38, 01187, Dresden, Germany, EU
}

(Dated: November 14, 2018)

\begin{abstract}
We investigate chaos in mixed-phase-space Hamiltonian systems using time series of the finitetime Lyapunov exponents. The methodology we propose uses the number of Lyapunov exponents close to zero to define regimes of ordered (stickiness), semi-ordered (or semi-chaotic), and strongly chaotic motion. The dynamics is then investigated looking at the consecutive time spent in each regime, the transition between different regimes, and the regions in the phase-space associated to them. Applying our methodology to a chain of coupled standard maps we obtain: (i) that it allows for an improved numerical characterization of stickiness in high-dimensional Hamiltonian systems, when compared to the previous analyses based on the distribution of recurrence times; (ii) that the transition probabilities between different regimes are determined by the phase-space volume associated to the corresponding regions; (iii) the dependence of the Lyapunov exponents with the coupling strength.
\end{abstract}

PACS numbers: $05.45 . \mathrm{Ac}, 05.45 . \mathrm{Pq}$

Keywords: Stickiness, finite-time Lyapunov spectrum, Poincaré recurrences, chaotic dynamics.

\section{INTRODUCTION}

In weakly chaotic Hamiltonian systems regions of regular (periodic and quasi-periodic) and chaotic motion typically coexist in the phase-space [1, 2]. In high dimensions, due to Arnold diffusion, all initial conditions leading to chaotic motion are connected in the phase-space building a single chaotic component [1]. Even if the volume of the regular regions becomes vanishingly small, as expected for high-dimensional nonlinear systems, the dynamics inside the chaotic component of the phase-space is strongly affected by such regions. This happens because trajectories approaching non-hyperbolic regions or regular motion remain a long time close to them before visiting again other parts of the chaotic component of the phase-space. This signature of weak mixing (or weak chaos) is known as stickiness [3 8].

Since Chirikov-Shepelyansky [3], the main quantification of stickiness in Hamiltonian systems has been through the fat-tail distribution of Poincaré recurrence times (see, e.g., [4, 8, 9]). An alternative approach is to use finite-time Lyapunov exponents (FTLEs) 1012], with recent applications using large deviation techniques [7, 13] and the cumulants [14, 15] of the FTLE distribution. In area-preserving maps, stickiness generically occurs at the border of 2-dimensional KolmogorovArnold-Moser (KAM) island [1] (i.e., at 1-dimensional tori). The recurrence time is a measure of the time the trajectory spends around such structures before returning to the chaotic sea (stickiness happens also to oneparameter families of parabolic orbits [16, 17] and even to isolated parabolic fixed points [4, 18, 19]). Near the non-hyperbolic structures, the local instability of chaotic trajectories is reduced so that FTLEs can be used to characterize phase-space regions of interest [11 13, 20, 21].
Stickiness has been studied also in higher-dimensional systems $9,10,14,15,21,22$, long recurrence times can be due to different non-hyperbolic regions and tori of different dimensionalities [23]. An improved characterization of stickiness events (long recurrence time) requires thus to measure the number of stable and unstable directions in the trajectory during this event. Froeschlé conjectured that lower-dimensional tori could not exist 1, 24, 25]. In early studies in the 80's such events of stickiness to lower dimensional tori were reported in some systems [21] but were not found in other examples [10]. Even if invariant tori do not exist, small local Lyapunov exponent could effectively act as a lowerdimensional trap. This is similar to almost invariant sets [26, 27], which are regions in phase-space where typical trajectories stay (on average) for long periods of time.

In this paper we introduce a methodology that uses time-series of local Lyapunov exponents to define regimes of ordered, semi-ordered and totally chaotic motion and obtain an improved characterization of stickiness in highdimensional Hamiltonian systems. We illustrate this general procedure in a chain of coupled standard maps and confirm that stickiness events of different times length are dominated by trajectories with different FTLEs. A significant improvement of the characterization of sticky motion in high-dimensional systems is found. We also characterize the FTLEs for small couplings and compare them to expected universal properties in fully chaotic systems [28]. The method proposed here is general and can be used to investigate Hamiltonian systems in any dimension.

The paper is divided as follows. In Sec. II we describe the Hamiltonian model we use to illustrate our method. In Sec. III we introduce our method to compute and analyze time series of local Lyapunov exponents. This methodology is then applied to the symplectic model of 
coupled standard maps in Sec. IV] Section V] summarizes the main results of the paper.

\section{THE COUPLED MAPS MODEL}

We use a time-discrete $2 N$-dimensional Hamiltonian system obtained as the composition $\mathbf{T} \circ \mathbf{M}$ of independent one-step iteration of $N$ symplectic 2-dimensional maps $\mathbf{M}=\left(M_{1}, \ldots, M_{N}\right)$ and a symplectic coupling $\mathbf{T}=\left(T_{1}, \ldots, T_{N}\right)$. As a representative example of 2 dimensional maps we choose for our numerical investigation the standard map:

$$
\mathbf{M}_{\mathbf{i}}\left(\begin{array}{l}
p_{i} \\
x_{i}
\end{array}\right)=\left(\begin{array}{ll}
p_{i}+K_{i} \sin \left(2 \pi x_{i}\right) & \bmod 1 \\
x_{i}+p_{i}+K_{i} \sin \left(2 \pi x_{i}\right) & \bmod 1
\end{array}\right)
$$

and for the coupling

$$
\mathbf{T}_{\mathbf{i}}\left(\begin{array}{l}
p_{i} \\
x_{i}
\end{array}\right)=\left(\begin{array}{l}
p_{i}+\sum_{j=1}^{N} \xi_{i, j} \sin \left[2 \pi\left(x_{i}-x_{j}\right)\right] \\
x_{i}
\end{array}\right)
$$

with $\xi_{i, j}=\xi_{j, i}=\frac{\xi}{\sqrt{N-1}}$ (all-to-all coupling). The motivation for working with this system is that in the limit of small coupling $\xi \rightarrow 0$ it can be understood looking at the dynamics of the $N$ uncoupled maps. This system was studied in Refs. [6, 9] using recurrence time distribution. This allow us to critically compare the benefits of our methodology. In all numerical simulations we used $K_{1}=0.5214$ for the map $M_{1}$ and $K_{2}=K_{3}=0.5108$ for the maps $M_{2}$ and $M_{3}$.

\section{METHOD}

In this section we describe the method proposed in this work. To be illustrative, we present numerical simulations for the system defined in Sec. II.

A. Lyapunov spectrum and the classification of ordered, semi-ordered or semi-chaotic, and chaotic regimes

Consider a chaotic trajectory in a closed Hamiltonian system which, after reducing the phase-space dimension due to global invariant of motion, has $N$ degree of freedoms. For long times $t$ the trajectory ergodically fills the whole chaotic component of the phase-space which is characterized by a spectrum of $N$ Lyapunov exponents $\left\{\lambda_{i=1 \ldots N}^{(\infty)}\right\}$, where $\lambda_{1}^{(\infty)}>\lambda_{2}^{(\infty)}, \ldots, \lambda_{N}^{(\infty)}>0$ [29]. The central ingredient of our analysis is the spectrum of FTLEs computed along a trajectory during a window of size $\omega$ where we obtain a time dependent spectrum $\left\{\lambda_{i}^{(\omega)}\right\}(t)=\left\{\lambda_{i}^{(\omega)}\right\}$. The window size $\omega$ has to be sufficiently small to guarantee a good resolution of the temporal variation of the $\lambda_{i}^{(\omega)}$ 's, but sufficiently large in order to have a reliable estimation (see Refs. 10 12]). The probability density function of $\lambda_{i}^{(\omega)}$ has been extensively studied [7, 10 12]. Here we go beyond the study of the probability density function and explore temporal properties in the time series of $\left\{\lambda_{i}^{(\omega)}\right\}$.

Figures 1(a) and (b), for $N=2$ and 3 respectively, show the time series of $\lambda_{i}^{(\omega)},(i=1, \ldots, N)$. The sharp transitions towards $\lambda_{i}^{(\omega)} \approx 0$ motivates the classification in regimes of motion [20, 21] as (a) ordered $\left(\lambda_{1,2}^{(\omega)} \approx 0\right)$; (b) semi-ordered or semi-chaotic $\left(\lambda_{1}^{(\omega)}>0 ; \lambda_{2}^{(\omega)} \approx 0\right)$; and (c) chaotic $\left(\lambda_{1,2}^{(\omega)}>0\right)$. For a system with $N$ degrees of freedom we will say that the trajectory is in a regime of type $S_{M}^{(N)}$ if it has $M$ local Lyapunov exponents $\lambda_{i}^{(\omega)}>\varepsilon_{i}$, where $\varepsilon_{i} \ll \lambda_{i}^{(\infty)}$ are the small thresholds. This means that $S_{0}^{(N)}$ and $S_{N}^{(N)}$ are ordered and chaotic regimes respectively. Whenever there is no ambiguity, we will drop the superscript $S_{M}^{(N)}=S_{M}$ to have a simpler notation.

Practical implementations of the general method described above require the choice of a few parameters and conventions. First of all, the window size $\omega$ and the threshold $\varepsilon_{i}$ directly affect the classification in regimes. They can be thought as the phase-space resolution of the analysis and should be chosen so that it provides maximal information about the regions of interest. Unless stated otherwise, we use $\omega=100$ and $\varepsilon_{i} \approx 0.10\left\langle\lambda_{i}^{(\omega)}\right\rangle$, where $\langle\ldots\rangle$ denotes average over $t$, where $t=1, \ldots, t_{L}$ (even though the classification in regimes is strongly $\omega$ dependent, our conclusions are not sensitively affected by variations around the chosen values). Another important choice is the method for computation of the FTLEs. We use Benettin's algorithm 30, 31], which includes the Gram-Schmidt re-orthonormalization procedure. The decreasing order of $\lambda_{i}^{(\omega)}$ is valid on average, but inversions of the order $\left(\lambda_{i+1}^{(\omega)}>\lambda_{i}^{(\omega)}\right)$ may happen for some times $t$ and we have chosen to impose the order of $\lambda_{i}^{(\omega)}$ for all $t$. Finally, it is possible to decide how to sample the time series $\lambda_{i}^{(\omega)}$. While the FTLEs are defined for all $t$, there is a trivial correlation between the values of FTLEs inside a window of size $\omega$ because they are computed using the same points of the trajectory. In order to avoid this trivial correlation the series of $\lambda_{i}^{(\omega)}$ can be computed using non-overlapping windows, i.e. plotting $\lambda_{i}^{(\omega)}$ only every $\omega$ time steps (a choice we adopt in our simulations).

\section{B. Identifying phase-space regions}

In order to understand the properties of the time series $\left\{\lambda_{i}^{(\omega)}\right\}$ it is useful to consider the phase-space regions associated to each regime $S_{M}$. We denote by $\mu(A)$ the phase-space volume (Liouville measure) of region $A$ in the 

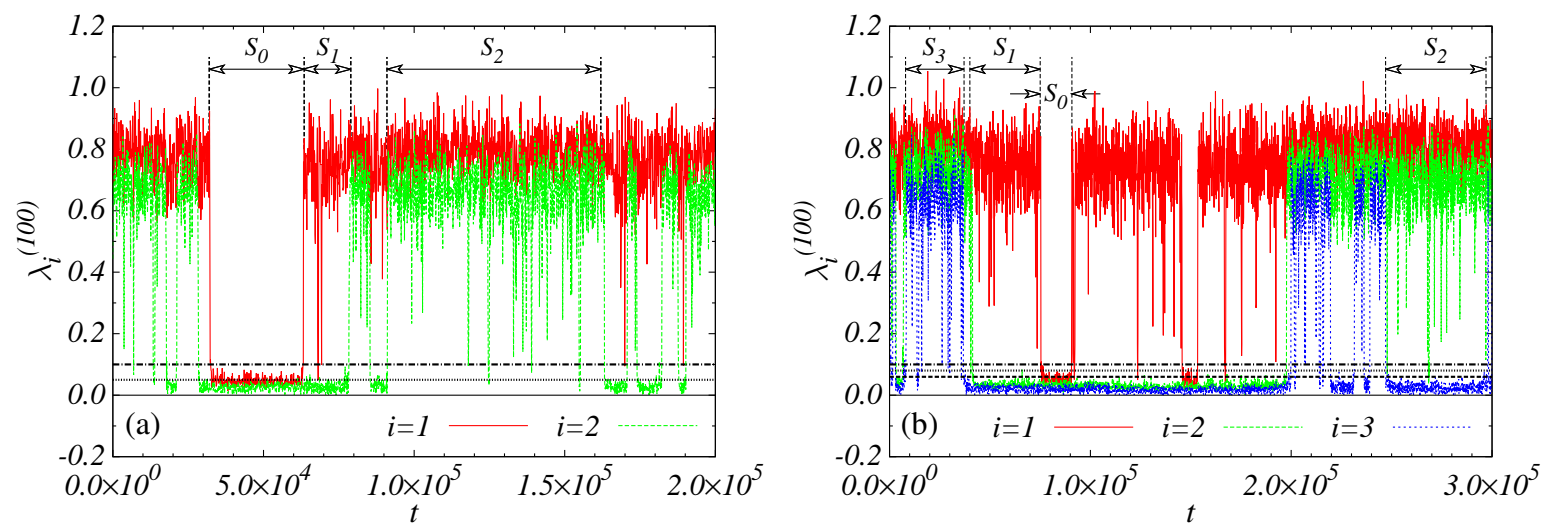

Figure 1. (Color online) Illustration of the method proposed to define the regimes $S_{M}$. Time series of the spectrum of FTLEs $\left\{\lambda_{i}^{(\omega=100)}\right\}$, with $i=1, \ldots, N$, for the map (11)-(2) with $\xi=10^{-3}$. Panel (a): Case $N=2$ and the thresholds $\varepsilon_{1}=0.1$ and $\varepsilon_{2}=0.05$ are represented by dash-dotted and dotted lines respectively. Panel (b): Case $N=3$ and the thresholds $\varepsilon_{1}=0.1$, $\varepsilon_{2}=0.08$ and $\varepsilon_{3}=0.06$ are represented by dash-dotted, dotted and dashed lines respectively.

bounded phase-space $\Gamma$, i.e. $\mu(\Gamma) \equiv 1$. The most important distinction is between the regions of regular $\Gamma_{\text {regular }}$ and chaotic $\Gamma_{\text {chaos }}$ motion. In Hamiltonian systems, typically $\mu\left(\Gamma_{\text {chaos }}\right)>0$ and $\mu\left(\Gamma_{\text {regular }}\right)>0$. In principle, the regular region $\Gamma_{\text {regular }}$ can be subdivided according to the dimensionality of the tori. However, according to Froeschlé's conjecture, in a $2 N$-dimensional phasespace, tori with dimension $N$ have positive measure and thus $\mu\left(\Gamma_{\text {regular }}\right)=\mu\left(\Gamma_{\text {tori }}\right)[1,24,25]$. For $N>1$, the chaotic region $\Gamma_{\text {chaos }}$ is expected to build a single ergodic component because tori of $N$ dimension do not partition the $2 N$-dimensional phase-space in different regions and therefore any chaotic trajectories eventually explores (through Arnold Diffusion) the whole $\Gamma_{\text {chaos }}$. Our interest is not to test the Froeschlé conjecture or Arnold diffusion, but to show the insights about the chaotic dynamics we can obtain using the time series of $\left\{\lambda_{i}^{(\omega)}\right\}$ together with the definition of the regimes $S_{M}$. One application is to use the regimes $S_{M}$ to split the chaotic component of the phase-space in meaningful components. This is done by considering the set of points $\boldsymbol{X}_{M}^{(N)}$ in the phase-space leading to each regime $S_{M}$ as

$$
\boldsymbol{X}_{M}^{(N)}=\lim _{t_{L} \rightarrow \infty} \boldsymbol{x}_{t}\left(\boldsymbol{x}_{t} \in S_{M}\right)
$$

where $t_{L}$ is the total length of the trajectory and $\boldsymbol{x}_{t} \in S_{M}$ indicates that at time $t$ the trajectory at $\boldsymbol{x}_{t}$ had $\left\{\lambda_{i}^{(\omega)}\right\} \in$ $S_{M}$.

Figure 2 shows numerical estimates of the phase-space regions obtained for each regime $S_{M}$ in the chain of coupled maps defined in Sec. III The regime $S_{0}$ (or the ordered regime) is associated to region localized close to the border of the KAM island of the uncoupled case (compare to Fig. 2a ). Points which belong to the regime $S_{1}$ are closer to the center of the torus from the uncoupled case. This suggests that when trajectories are inside the region related to regime $S_{1}$, they more likely penetrate inside the torus from the uncoupled case. In the chaotic sea both regimes $S_{1}$ and $S_{2}$ are visible. These results are naturally understood in the perturbative limit (small coupling $\xi \ll 1)$. The regime $S_{0}$ corresponds to $\lambda_{i}^{(100)} \approx 0$ for every $i=1, \ldots, N$, which is expected when the trajectory is stuck close to the $N$-dimensional tori built as the product of the 1-dimensional tori of the uncoupled maps. In contrast, $S_{M}$ for $M>0$ implies that at least one FTLE $\lambda_{i}^{(\omega)} \gg 0$ and therefore the trajectory projected in one map can be both in the chaotic and regular regions (e.g., $S_{1}$ for $N=2$ can be obtained from $\lambda_{1}^{(\omega)} \gg 0, \lambda_{2}^{(\omega)} \approx 0$ or from $\left.\lambda_{1}^{(\omega)} \approx 0, \lambda_{2}^{(\omega)} \gg 0\right)$. Altogether, these observations confirm that our method allows for a meaningful division of the chaotic component of the phase-space and can thus be used to identify regions of interesting dynamics. In the case partial barriers exist inside the chaotic component - such as in area-preserving maps with mixed phase space [2] - we expect the regions obtained through our method to depend weakly on $\omega$ and to coincide with those obtained from the partial barriers.

\section{RESULTS}

In this Section we apply the Lyapunov time-series methodology described in Sec. III to the $2 N$-dimensional system defined in Sec. [II. We compute and interpret four basic properties of the method: the total time spent in each regime (residence time), the transition between regimes, the consecutive time in each regime, and the scaling of Lyapunov exponents.

\section{A. Residence time in each regime}

The first and most basic quantity we measure is the probability $P\left(S_{M}\right)$ of finding the trajectory in each 

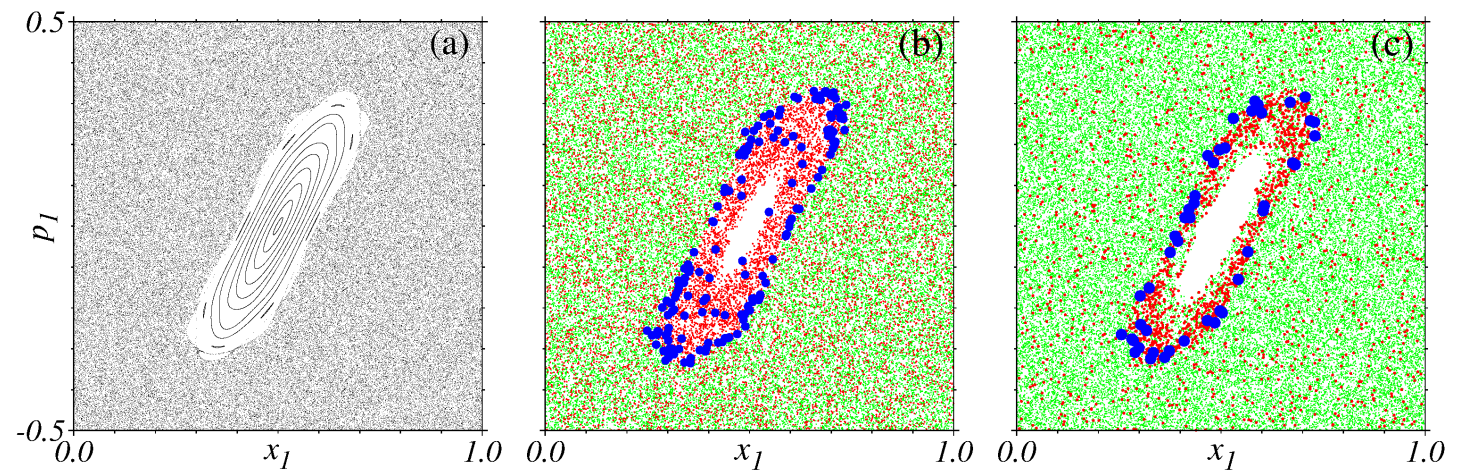

Figure 2. (Color online) Phase-space projected in $\left(x_{1}, p_{1}\right)$ for different configuration of the $N$-coupled standard maps defined in Sec. III (a) $N=1$ (uncoupled case), showing $10^{2}$ randomly started initial conditions and plotting as dots $10^{4}$ iterations of each of them. A large KAM island can be seen at the center of the plot; (b) $N=2$ and coupling strength $\xi=10^{-3}$; (c) $N=3$ and $\xi=10^{-3}$. Symbols with different colors in (b,c) show points $\boldsymbol{x}_{t} \in S_{M}$ belonging to regimes $S_{0}$ (blue circles), $S_{1}$ (red points), and $S_{2}$ (green points). These points were computed starting a single trajectory in the chaotic region of all maps and iterating it $5 \times 10^{6}$ times.

regime, defined as the fraction of the total time $t_{L}$ that $\boldsymbol{x}_{t} \in S_{M}$ (i.e. $P\left(S_{M}\right)=\sum_{t=0}^{t_{L}} \delta_{t \in S_{M}} / t_{L}$, where $\delta_{t \in S_{M}}=1$ if $t \in S_{M}$ and $\delta_{t \in S_{M}}=0$ otherwise).
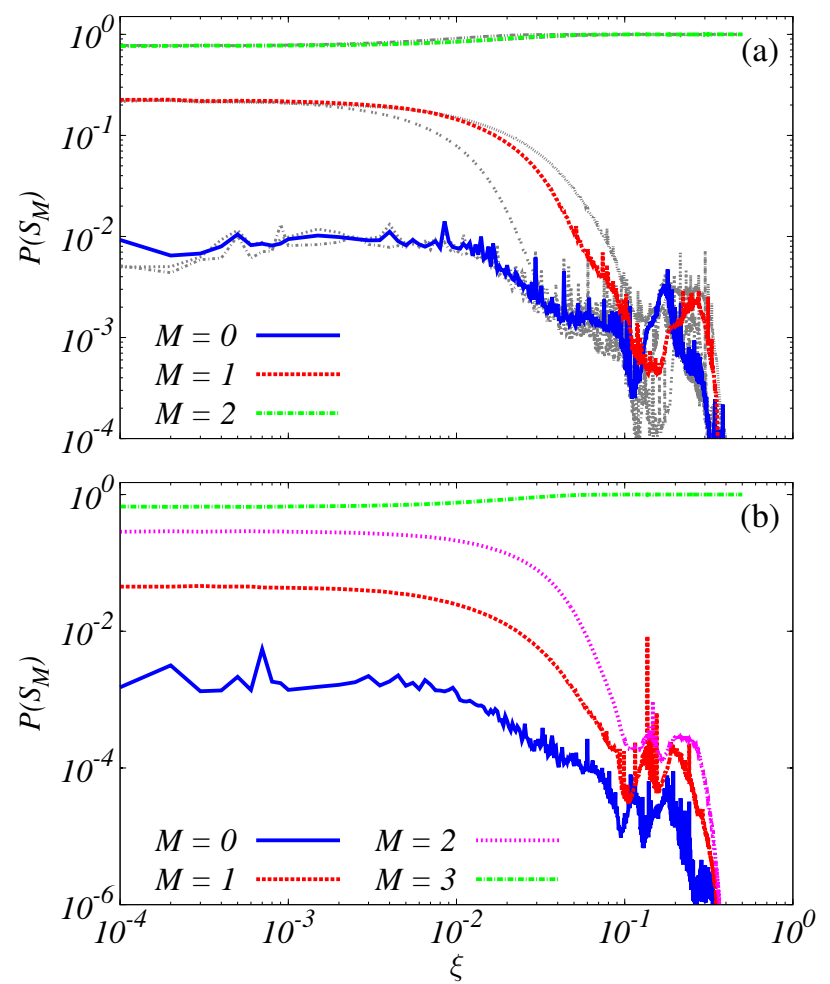

Figure 3. (Color online) Residence time in each regime $S_{M}$. (a) $N=2$ with $\varepsilon_{1}=0.1$ and $\varepsilon_{2}=0.05$. (b) $N=3$ with $\varepsilon_{1}=0.1, \varepsilon_{2}=0.08$, and $\varepsilon_{3}=0.06$. In (a) the values obtained with $\omega=100$ are compared (gray curves) with results for $\omega=50$ and $\omega=500$. Only for the case $M=1$ the gray curves (right for $\omega=50$ and left for $\omega=500$ ) show a shift in the $x$-axis $\xi$. Estimations for each $\xi$ are based on a trajectory with length $t_{L}=10^{10}$.
Figure 3 shows the probabilities $P\left(S_{M}\right)$ for the map with $N=2,3$ as a function of the coupling strength $\xi$. We now explain the behavior of $P\left(S_{M}\right)$ with $\xi$ by discussing the effect of coupling $\xi$ on the phase-space regions associated to $S_{M}$, as defined in Sec. IIIB. By the ergodicity of $\Gamma_{\text {chaos }}, P\left(S_{M}\right)$ corresponds to the (normalized) volume of the region related to regime $S_{M}$ in the phase-space

$$
P\left(S_{M}\right)=\frac{\mu\left(S_{M}\right)}{\mu\left(\Gamma_{\text {chaos }}\right)}=\frac{\mu\left(S_{M}\right)}{1-\mu\left(\Gamma_{\text {tori }}\right)} .
$$

The results of Fig. 3 show that the chaotic region is the largest region in phase-space for any coupling, while the region associated to $S_{1}$ has a larger volume than $S_{0}$ for couplings $\xi \lesssim 1.3 \times 10^{-1}$. For larger $\xi$ we see oscillations with a local maximum close to $\xi \sim 2 \times 10^{-1}$ for the cases $M=0$ and $M=1$.

We now interpret the $\xi$ dependence observed in Fig. 3 by arguing how the different terms in Eq. (4) vary with $\xi$. We denote by $\mu\left(U_{j}\right)$ the measure of tori for the $j$-th map with control parameter $K_{j}$ in the uncoupled case $\xi=0$ (which we assume to be approximately equal to the measure of the KAM islands). For small coupling $\xi \approx 0$ we expect that most tori of the uncoupled maps to survive and therefore:

- $\mu\left(\Gamma_{\text {tori }}\right) \approx \prod_{j=1}^{N} \mu\left(U_{j}\right)$, which in the simple case of $\mu\left(U_{j}\right)=\mu$ for all $j$ reduces to $\mu\left(\Gamma_{\text {tori }}\right) \approx \mu^{N}$.

- $\mu\left(S_{M=0}\right)$ corresponds to a small volume around $\Gamma_{\text {tori }}$, i.e. $\mu\left(S_{M=0}\right) \sim \mu\left(\Gamma_{\text {tori }}\right) \approx P\left(S_{0}\right) /(1+$ $\left.P\left(S_{0}\right)\right)$.

- For $\mu\left(S_{M \neq 0}\right)$ we have that $N-M$ maps are in their corresponding KAM island (with probability $\left.\mu\left(U_{j}\right)\right)$ and $M$ maps in the chaotic area (with probability $\left.1-\mu\left(U_{k}\right)\right)$. For example, for $N=3$ and $M=2$ we have that

$$
\mu\left(S_{M=2}\right)=\mu\left(U_{1}\right)\left[1-\mu\left(U_{2}\right)\right]\left[1-\mu\left(U_{3}\right)\right]
$$




$$
\begin{aligned}
& +\mu\left(U_{2}\right)\left[1-\mu\left(U_{1}\right)\right]\left[1-\mu\left(U_{3}\right)\right] \\
& +\mu\left(U_{3}\right)\left[1-\mu\left(U_{1}\right)\right]\left[1-\mu\left(U_{2}\right)\right] .
\end{aligned}
$$

In general this leads to

$$
\mu\left(S_{M}\right) \approx \sum_{j_{1}} \ldots \sum_{j_{M}} \prod_{j \in\left\{j_{1}, \ldots, j_{M}\right\}}\left(1-\mu_{j}\right) \prod_{j \notin\left\{j_{1}, \ldots, j_{M}\right\}} \mu_{j},
$$

where the last product is over all $j=1, \ldots, N$ except $j \in\left\{j_{1}, \ldots, j_{M}\right\}$. In the simple case of $\mu\left(U_{j}\right)=\mu$, it reduces to $\mu\left(S_{M}\right) \approx\left(\begin{array}{l}N \\ M\end{array}\right) \mu^{N-M}(1-$ $\mu)^{M}$.

We now consider the effect of growing $\xi$. In the spirit of the KAM theorem, the tori of the coupled maps (generated as the product of the $N$ maps) are expected to be robust to small couplings $\xi$, which act as a perturbation. This explains why the curves in Fig. 3 are essentially flat for small $\xi$. Increasing $\xi$ even further, the nonlinearity of the system increases and therefore $\mu\left(\Gamma_{\text {tori }}\right)$ is expected to decrease $\left(\mu\left(\Gamma_{\text {tori }}\right) \rightarrow 0\right.$ for $\left.\xi \gg 0\right)$. This reduction of the tori leads to an increase in the denominator of Eq. (4) and explains the observed tendency of reduction of $P\left(S_{M}\right)$ for all regions related to stickiness $(M<N)$. Indeed, for $\xi>0.5$ no signature of tori or stickiness was detected numerically and $P\left(S_{M=N}\right)=1$. The nontrivial dependencies of $P\left(S_{M<N}\right)$ in Fig. 3 appear at $\xi \sim 2 \times 10^{-1}$ values, close to the values of $\xi$ for which the last tori disappear (see also Fig. 7.2 in Ref. [6]). In this regime the volume of the tori is already negligible $\mu\left(\Gamma_{\text {tori }}\right) \gtrsim 0$ but stickiness is still effective (notice that even zero measure non-hyperbolic sets can lead to stickiness [7, 17]). The denominator in Eq. (4) is therefore $1-\mu\left(\Gamma_{\text {tori }}\right) \sim 1$, not significantly affected by further increases of $\xi$, and therefore not driving the reduction of $P\left(S_{M<N}\right)$. Small variation of a control parameter of the system (in this case $\xi$ ) are known to lead to sensitive creation and destruction of tori, with non-trivial dependencies on the stickiness [5]. We can thus expect that close to the disappearance of the tori - the small volume of stickiness regions $\mu\left(S_{M<N}\right)$ to fluctuate with $\xi$ leading even to an increase with $\xi$. It is interesting to note that this non-trivial increase with $\xi$ appears for $P\left(S_{M=0}\right)$ in Fig. 3 precisely when the curves $P\left(S_{0<M<N}\right)$ show a sharp decreasing fluctuation. This suggests an exchange between measure of different sticky regions associated to regimes $S_{M<N}$, without interference of the much larger fully chaotic component $S_{M=N}$.

\section{B. Transitions between regimes}

We now focus on the transition between regimes. The simplest analysis correspond to the two-time (joint) probability $P\left(S_{M} \rightarrow S_{M^{\prime}}\right)$, computed as the fraction of the total trajectory time $t_{L}$ that $\boldsymbol{x}_{t} \in S_{M}$ and $\boldsymbol{x}_{t+1} \in S_{M^{\prime}}$. The probabilities considered in the previous section can be obtained as $\sum_{S_{M}} P\left(S_{M} \rightarrow S_{M^{\prime}}\right)=P\left(S_{M^{\prime}}\right)$ and $\sum_{S_{M^{\prime}}} P\left(S_{M} \rightarrow S_{M^{\prime}}\right)=P\left(S_{M}\right)$. Figure 4(a) shows the
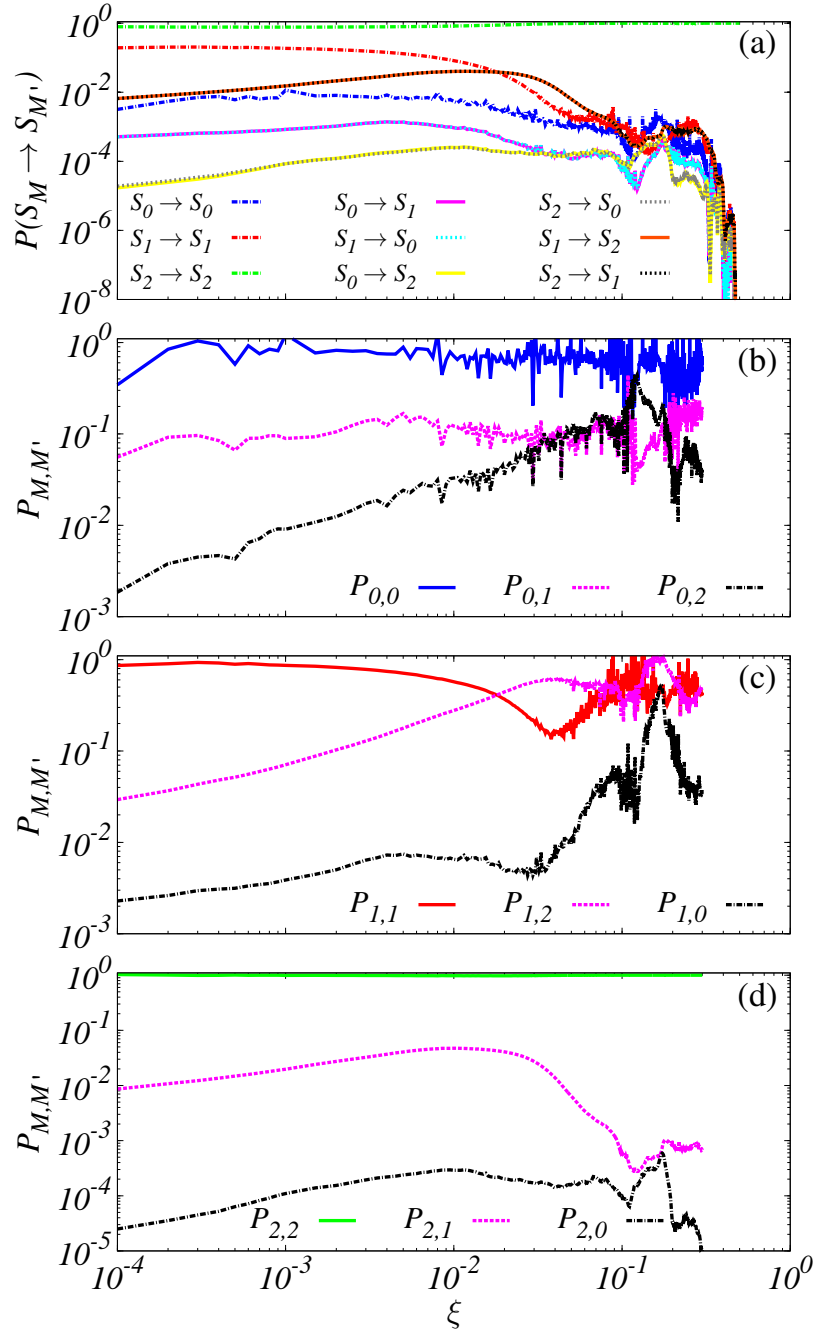

Figure 4. (Color online) Transition between regimes $S_{M}$ as a function of the coupling strength $\xi$. (a) Transition probability $P\left(S_{M} \rightarrow S_{M^{\prime}}\right)$; (b-d) Conditional probability $P_{M, M^{\prime}}$ defined in Eq. (5) of moving to $M^{\prime}$ given that the trajectory was at $M$. Estimations for each $\xi$ based on a single trajectory with length $t_{L}=10^{10}$ in the case of $N=2$ coupled maps and $\varepsilon_{1}=0.1$ and $\varepsilon_{2}=0.05$.

dependence of $P\left(S_{M} \rightarrow S_{M^{\prime}}\right)$ on $\xi$ for our model. We notice that $P\left(S_{M} \rightarrow S_{M^{\prime}}\right)$ is equal to $P\left(S_{M^{\prime}} \rightarrow S_{M}\right)$. This is expected considering that the system is ergodic, volume preserving, and time-reversible. The dependence of $P\left(S_{M} \rightarrow S_{M^{\prime}}\right)$ on $\xi$ follows a similar pattern observed for $P\left(S_{M}\right)$ in Fig. 3. More information is obtained from the conditional probability

$$
P_{M, M^{\prime}} \equiv P\left(S_{M} \rightarrow S_{M^{\prime}} \mid S_{M}\right) \equiv \frac{P\left(S_{M} \rightarrow S_{M^{\prime}}\right)}{P\left(S_{M}\right)},
$$

which quantifies the probability that trajectories at $S_{M}$ will move to $S_{M^{\prime}}$. The results shown in Fig. 4(b-d) show for all $S_{M}$ that (i) persistence in the same $S_{M}\left(P\left(S_{M} \leftrightarrow\right.\right.$ $\left.S_{M} \mid S_{M}\right)$ ) is dominant and (ii) the most likely transitions occur between neighboring regimes (e.g., $\left.P_{2,1}>P_{2,0}\right)$. 
The only (slight) deviations of this picture happen for large values of $\xi$, close to the disappearance of the KAM island. Altogether, these results confirm that in the perturbative regime $(\xi \ll 1)$ stickiness happens approaching the region of regular motion of different maps one after the other (in opposite to a direct approach from $S_{0}$ to $\left.S_{M=N}\right)$.

\section{Consecutive time in each regime}

The results of the previous section confirm that residence in the same regime is the dominant behavior. This motivates us to study the time $\tau_{M}$ spent consecutively in a regime $S_{M}$ (i.e., $\tau_{M}$ is the time between two consecutive transitions between different regimes, the first to $S_{M}$ and the second out of $S_{M}$ ). In a trajectory of length $t_{L}$ we collect a series of $\tau_{M}$. We are mainly interested in the probability distribution $P\left(\tau_{M}\right)$ (or, equivalently its cumulative $P_{\text {cum }} \equiv \sum_{\tau_{M}^{\prime}=\tau_{M}}^{\infty} P\left(\tau_{M}^{\prime}\right)$ ) for different $S_{M}$ 's in the limit $t_{L} \rightarrow \infty$. These distributions should be compared to the distribution of recurrence times $\tau$, defined as the time between two successive entries to a pre-defined recurrence region (usually taken in the fully chaotic component of the phase-space). Events in the tails of $P(\tau)$ are associated to times for which the trajectory is stuck to the non-hyperbolic components of the phase-space and $P(\tau)$ is the traditional method to quantify stickiness in Hamiltonian systems [3, 4, 8, 9].

The numerical simulations in Fig. 5] confirm that the distribution obtained summing $P_{\text {cum }}\left(\tau_{M}\right)$ for ordered and semi-ordered regimes (or $S_{M<N}$ ) is equivalent to cumulative distribution $P_{\text {cum }}(\tau)$ obtained using recurrences. This is in agreement with the association of long consecutive times in regimes of ordered and semiordered motion to long recurrence times. Looking at the individual distributions $P_{\text {cum }}\left(\tau_{M}\right)$ provide valuable additional information on the sticky motion. For semiordered motion (when $0<M<N$ ) we observe an exponential tail after an intermediate decay with scaling $\beta \approx 0.5$. This behavior confirms the interpretation given in Ref. [9]. More interestingly, the $M=0$ case shows an asymptotic algebraic decay which characterize stickiness. While the scaling is compatible with the results obtained using recurrence time, $P_{c u m}\left(\tau_{M=0}\right)$ obtained in our methodology provides a better characterization of the scaling (over several orders of magnitudes) and allows for an independent analysis of the different regimes. These properties are essential when dealing with highdimensional systems (which may contain different preasymptotic regimes) and for an accurate estimation of the stickiness exponent $\gamma$. Finally, panel (a) in Fig. 5 shows that all decays discussed above remain (qualitatively) the same for different choices of $\omega$, with the curve for $M=1$ showing the largest sensitivity on $\omega$ (as in Fig. [3(a).
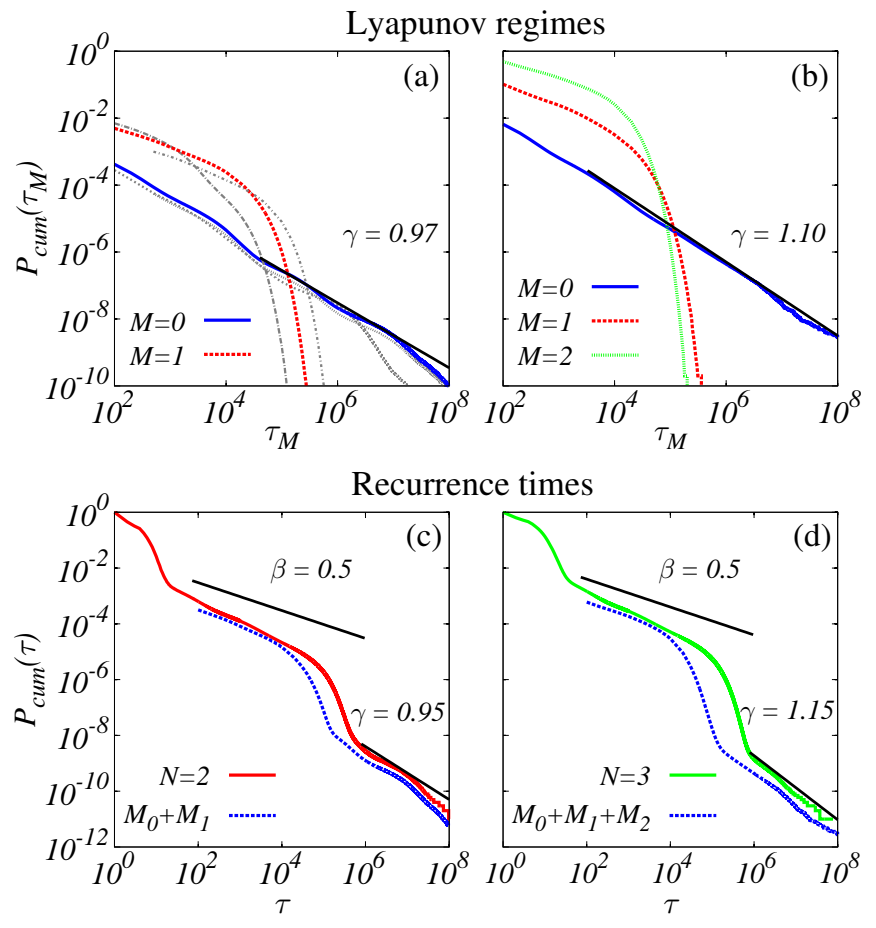

Figure 5. (Color online) Comparison between our method and the analysis based on recurrence time. The cumulative distribution $P_{\text {cum }}\left(\tau_{M}\right)$ of times $\tau_{M}$ is shown for each regime $S_{M<N}$ for $\omega=100$ and (a) $N=2$ and (b) $N=3$. In (a) the gray curves show results for $\omega=50$ and $\omega=500$. Only for the case $M=1$ the gray curves (left for $\omega=50$ and right for $\omega=500)$ show a shift in the $x$-axis $\xi$. The cumulative distribution $P_{\text {cum }}(\tau)$ for recurrence times $\tau$ to a region in the chaotic component of the phase-space (in $S_{M=N}$ ) for (c) $N=2$ and (d) $N=3$. For comparison, in panels (c) we show the results obtained combining the normalized curves for $M_{0}+M_{1}$ (blue dotted line: divided by $1.7 \times 10^{3}$ for convenience of scale) of panel (a), and in (d) the normalized curves for $M_{0}+M_{1}+M_{2}$ (blue dotted line: divided by $10^{3}$ ) of panel (b). Results obtained using maps of Sec. II with $\xi=10^{-3}, \varepsilon_{1}=0.1$ and $\varepsilon_{2}=0.05$ for the case $N=2$ and $\varepsilon_{1}=0.1, \varepsilon_{2}=0.08$ and $\varepsilon_{3}=0.06$ for the case $N=3$.

\section{Scaling of Lyapunov exponents}

So far we have focused at the temporal properties of the time series of FTLEs $\lambda_{i}^{(\omega)}$ and how they change with the coupling strength $\xi$. We now consider how the value of the Lyapunovs respond to an external perturbation, which in our case is the coupling to the other maps. It is known that the largest exponent $\lambda_{1}^{(\infty)}$ is extremely sensitive to perturbation. More specifically, Daido's relation 28, 32] states that for small couplings $\xi$ to another chaotic system, a universal logarithmic singularity is observed,

$$
\lambda_{i}^{(\omega \rightarrow \infty)}-\Lambda_{i}^{(\omega \rightarrow \infty)} \approx \frac{c}{|\ln (\xi)|}
$$


where $\Lambda_{i}^{(\omega \rightarrow \infty)}$ are the unperturbed Lyapunov exponents and $c$ is a constant and $i=1, \ldots, N$. This relation is valid for totally chaotic systems and a small mismatch between Lyapunov exponents of the uncoupled systems compared to their fluctuations [32]. Here we investigate the relation $\lambda_{i}^{(\omega)}-\Lambda_{i}^{(\omega)}$ as a function of $\xi$, for distinct values of $\omega$ and different regimes $S_{M}$. To this end we compute the temporal averages of the FTLEs $\left\langle\lambda_{i}^{(\omega)}\right\rangle$ for times $t$ such that $\left\{\lambda_{i}^{(\omega)}\right\} \in S_{M}$.
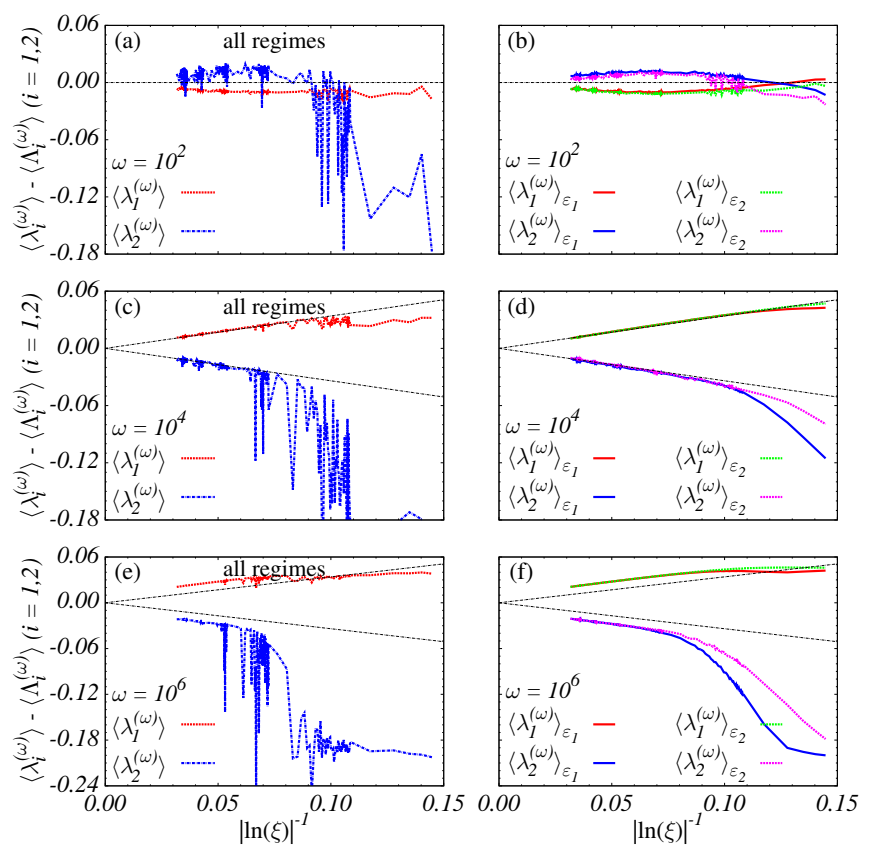

Figure 6. (Color online) Sensitivity of the FTLEs for small couplings. The difference between the finite-time Lyapunov exponent in the coupled $\left(\left\langle\lambda_{i}^{(\omega)}\right\rangle\right)$ and uncoupled $\left(\left\langle\Lambda_{i}^{(\omega)}\right\rangle\right)$ maps as a function of $|\ln (\xi)|^{-1}$, where $\xi$ is the coupling strength. Results are shown for $N=2(i=1,2)$ and different time windows $\omega$ (a)-(b) $10^{2}$, (c)-(d) $10^{4}$, and (e)-(f) $10^{6}$. Black dashed lines in (c)-(f) are the expected linear behaviour, consistent with Eq. (6). Panels in the left column (ace) were computed for the full time series, while on the right column (bdf) only FTLEs in the regime $S_{2}$ were used. The different colors correspond to different choices of threshold imposed to define the FTLE: $\left\langle\lambda_{i}^{(\omega)}\right\rangle_{\varepsilon_{1}}$ uses $\varepsilon_{1}=0.1\left\langle\lambda_{i}^{(\omega)}\right\rangle$ while $\left\langle\lambda_{i}^{(\omega)}\right\rangle_{\varepsilon_{2}}$ uses $\varepsilon_{2}=0.9\left\langle\lambda_{i}^{(\omega)}\right\rangle$, where $\left\langle\lambda_{i}^{(\omega)}\right\rangle$ is computed over the full time series (left column).

Our numerical simulations reported in Fig. 6] show that small values of $\omega$ lead to a situation in which $\left\langle\lambda_{i}^{(\omega)}\right\rangle \approx\left\langle\Lambda_{i}^{(\omega)}\right\rangle$ at a finite value of $\xi$ (Figs. 6(b) and (d)), while larger values of $\omega$ lead to situations in which $\left\langle\lambda_{i}^{(\omega)}\right\rangle \neq\left\langle\Lambda_{i}^{(\omega)}\right\rangle$ for any $\xi$. These results depend crucially on our choice to impose the order of $\lambda_{i}^{(\omega)}$ for all $t$, as discussed in Sec. IIIA. This makes the average over the trajectory time $\left\langle\lambda_{i}^{(\omega)}\right\rangle$ to be $\omega$-dependent and different from the average over the Lyapunov time $\lambda_{i}^{(\omega \rightarrow \infty)}$. Applying the analysis without the division in regimes
$S_{M}$ leads to strongly fluctuating results (Figs. 6(a,c,e)). Much smoother results (Figs. 6 $(b, d, f))$ are obtained when we apply our method and compute $\left\langle\lambda_{i}^{(\omega)}\right\rangle$ only for $t$ in the fully chaotic regime $S_{N}$. Looking at these smoother results we observe that the difference in Lyapunovs scales as $1 /|\ln \xi|$, but that even for $\omega \rightarrow \infty$ the sticky motion leads to a deviation from Daido's relation (6) (curves are shifted vertically).

\section{CONCLUSIONS}

In summary, we have proposed a method to characterize the dynamics of Hamiltonian systems with mixed phase-space based on time series of finite-time Lyapunov exponents. Using this method it is possible to define and study with high accuracy the time evolution of regimes of ordered, semi-ordered, and totally chaotic motion. This allows for an individualized characterization of the different stickiness mechanisms, improving alternative methods based on the statistics of recurrence times or on the distribution of finite-time Lyapunov exponents.

We applied our method to a chain of coupled standard maps and showed how the frequency of different regimes - and the transition probabilities between them - are related to the volume of different phase-space regions. Using the consecutive time in distinct regimes we have reproduced previous results obtained using recurrence times and showed that our method allows for a significant improvement in the characterization of the sticky motion (e.g., in the determination of the scaling exponents). This indicates that our method can be used to characterize stickiness in general high-dimensional systems and is particularly suited for cases in which different regions of sticky motion coexist. We have also shown that the dependence on the coupling strength of the largest Lyapunov exponents, after conveniently using our procedure, tend to follow only the qualitative universal properties of fully chaotic system.

Results obtained in a simple chain of standard maps confirm that our methodology can be applied to highdimensional systems and problems of current interest, such as controlling Fermi acceleration 33], galactic models [34], and plasma physics [35]. Another example of application is to associate each regime $S_{M}$ with effective Hamiltonian functions, a procedure used to reproduce the complicated dynamics of kicking electrons [36] or the high harmonic generation in laser-assisted collisions [37].

\section{ACKNOWLEDGMENTS}

CM and RMS thank CNPq, CAPES and FAPESC and MWB thanks CNPq for financial support and MPIPKS in the framework of the Advanced Study Group on Optical Rare Events. CM also thanks Eduardo G. Altmann for the financial support and hospitality at the MPIPKS. 
EGA thanks D. Pazó for suggesting the analysis per-
[1] A. J. Lichtenberg and M. A. Lieberman, Regular and Chaotic Dynamics (Springer-Verlag, New York, 1992).

[2] J. D. Meiss, Rev. Mod. Phys. 64, 795 (1992).

[3] B. V. Chirikov and D. L. Shepelyansky, Physica D 13D, 395 (1984).

[4] R. Artuso, Physica D 131, 68 (1999).

[5] G. M. Zaslavsky, Physics Reports 371, 461 (2002).

[6] E. G. Altmann, Ph.D. thesis, Max Planck Institut für Physik Komplexer Systeme (2007).

[7] R. Artuso and C. Manchein, Phys. Rev. E 80, 036210 (2009).

[8] G. Cristadoro and R. Ketzmerick, Phys. Rev. Lett. 100, 184101 (2008).

[9] E. G. Altmann and H. Kantz, Europhys. Lett. 78, 10008 (2007).

[10] H. Kantz and P. Grassberger, Phys. Lett. A 123, 437 (1987).

[11] J. D. Szezech, S. R. Lopes, and R. L. Viana, Phys. Lett. A 335, 394 (2005).

[12] M. Harle and U. Feudel, Chaos, Solitons \& Fractals 31, 130 (2007).

[13] T. Laffargue, K.-D. N. T. Lam, J. Kurchan, and J. Tailleur, Journal of Physics A: Mathematical and Theoretical 46, 254002 (2013).

[14] C. Manchein, M. W. Beims, and J. M. Rost, Chaos 22, 033137 (2012).

[15] C. Manchein, M. W. Beims, and J. M. Rost, Physica A 400, 186 (2014).

[16] P. Gaspard and J. Dorfman, Phys. Rev. E 52, 3525 (1995).

[17] E. G. Altmann, A. E. Motter, and H. Kantz, Phys. Rev. E 73, 026207 (2006).

[18] R. Artuso and A. Prampolini, Phys. Lett. A 246, 407 (1998).

[19] M. Sala, C. Manchein, and R. Artuso, arXiv:1410.4806.

[20] G. Contopoulos, L. Galgani, and A. Giorgilli, Phys. Rev. A 18, 1183 (1978).
[21] A. Malagoli, G. Paladin, and A. Vulpiani, Phys. Rev. A 34, 1550 (1986).

[22] D. Mingzhou, T. Bountis, and E. Ott, Phys. Lett. A 151, 395 (1990).

[23] S. Lange, M. Richter, F. Onken, A. Bäcker, and R. Ketzmerick, Chaos 24, 024409 (2010).

[24] C. Froeschlé, Astrophys. Space Sci. 14, 110 (1971).

[25] C. Froeschlé, Astron. \& Astrophys. 16, 172 (1972).

[26] M. Dellnitz and O. Junge, Int. J. Bif. Chaos 7, 2475 (1997).

[27] G. Froyland and K. Padberg, Physica D 238, 1507 (2009).

[28] H. Daido, Prog. Theor. Phys. 72, 853 (1984).

[29] Note1, without loss of generality we focus on the $N$ largest Lyapunov exponents because due to the symplectic character of Hamiltonian systems the others $N$ exponents are simply $\lambda_{N+1}=-\lambda_{N}, \lambda_{N+2}=$ $-\lambda_{N-1}, \ldots \lambda_{2 N}=-\lambda_{1}$.

[30] G. Benettin, L. Galgani, A. Giorgilli, and J.-M. Strelcyn, Meccanica 15, 09 (1980).

[31] A. Wolf, J. B. Swift, H. L. Swinney, and J. A. Vastano, Physica D 16, 285 (1985).

[32] R. Zillmer, V. Ahlers, and A. Pikovsky, Phys. Rev. E 61, $332(2000)$.

[33] A. L. P. Livorati, T. Kroetz, C. P. Dettmann, I. L. Caldas, and E. D. Leonel, Phys. Rev. E 86, 036203 (2012).

[34] G. Contopoulos and M. Harsoula, Celest. Mech. Dyn. Astr. 107, 77 (2010).

[35] D. del Castillo-Negrete, B. A. Carreras, and V. E. Lynch, Phys. Rev. Lett. 94, 065003 (2005).

[36] M. Gerlach, S. Wüster, and J. M. Rost, J. Phys. B 45, $235204(2012)$

[37] C. Zagoya, C. M. Goletz, F. Grossmann, and J. M. Rost, New J. Phys. 14, 093050 (2012). 